\title{
REVEW
}

\section{Recent progress in low-order density matrix theory of inhomogeneous electron liquids by exact solution of two- and four-electron model atoms}

\author{
A. Akbari ${ }^{1}$, N. H. March ${ }^{2,3,4}$, A. Rubio ${ }^{1}$, G. G. N. Angilella ${ }^{5,6,7,8 *}$ R. Pucci ${ }^{5,6}$ \\ ${ }^{1}$ Nano-Bio Spectroscopy Group and ETSF Scientific Development Centre, \\ Departamento de Física de Materiales, Centro de Física de Materiales \\ CSIC-UPV/EHU-MPC and DIPC, Universidad del País Vasco UPV/EHU, \\ Avenida Tolosa 72, E-20018 San Sebastián, Spain \\ ${ }^{2}$ Department of Physics, University of Antwerp, \\ Groenenborgerlaan 171, B-2020 Antwerp, Belgium \\ ${ }^{3}$ Abdus Salam International Centre for Theoretical Physics, Trieste, Italy \\ ${ }^{4}$ Oxford University, Oxford, UK \\ ${ }^{5}$ Dipartimento di Fisica e Astronomia, Università di Catania, \\ Via S. Sofia, 64, I-95123 Catania, Italy \\ ${ }^{6}$ CNISM, UdR di Catania, Via S. Sofia, 64, I-95123 Catania, Italy \\ ${ }^{7}$ INFN, Sez. Catania Via S. Sofia, 64, I-95123 Catania, Italy \\ ${ }^{8}$ Scuola Superiore di Catania, Università di Catania, \\ Via Valdisavoia, 9, I-95123 Catania, Italy \\ (Received on ..., in final form)
}

\begin{abstract}
Three aspects of low-order density matrix (DM) theory will be reviewed, following some brief comments on analogies with, and differences from, density functional theory (DFT). First, the local energy equation, involving first-to-third order DMs, will be set out, and applied exactly to model spin-compensated two-electron atoms. Explicit relations known between low-order DMs for harmonic confinement and arbitrary interparticle interactions will be reported for such model atoms. Secondly, the March-Young proposal for use variationally, satisfying $N$-representability, will be set out for spin-free
\end{abstract}

${ }^{*}$ Corresponding author. E-mail: giuseppe.angilella@ct.infn.it 
systems such as a four-electron model in the quintet state. Thirdly, the equation of motion for the correlated 1DM is summarized, and brief comments made on its application to (a) the He atom, and (b) crystalline Si. Finally, a model two-electron atom with Coulomb confinement plus an $s$-wave Coulomb repulsion modified by a $\delta$-function radial correlation is reported as an exactly solvable example.

Keywords: Local energy equation; Moshinsky atom; Equation of motion of 1DM.

Running head: Density matrix theory of inhomogeneous electron liquids

\section{Background and outline}

After a brief summary of the Löwdin $[1,2,3]$ definitions of low-order density matrices (DMs), some brief comments will be made in relation to density functional theory (DFT) [4] (see especially Sec. 2 below). Then, following especially the account of Dawson and March [5], the local energy equation of DM theory will be set out for arbitrary external potential and general interaction $u\left(r_{12}\right)$. Though, in general, this involves $1-3 \mathrm{DMs}$, to our knowledge, at the time of writing, this local energy equation has only been solved analytically for two-electron spin compensated model atoms with harmonic confinement specifying the external potential.

So, as to the outline, Sec. 2 will quote the $1 \mathrm{DM}$ for such a two-electron model, but with harmonic interactions also, following [6]. One of us [7] obtained the 1DM $\gamma\left(\mathbf{r}, \mathbf{r}^{\prime}\right)$ for this model as a functional of its diagonal density $\rho(\mathbf{r})$, enabling the total correlated kinetic energy to be thereby obtained as a functional of the density $\rho$. Then, in Sec. 3, following the study of Holas, Howard, and March [8], $\gamma\left(\mathbf{r}, \mathbf{r}^{\prime}\right)$ is reported explicitly for arbitrary $u\left(r_{12}\right)$, the Moshinsky atom being, of course, contained as a special case, with the Hookean atom $[9,10]$ referred to as a further example.

More briefly, in Sec. 4, after a brief comment on the $N$-representability problem for variationally valid low-order density matrices for many-electron assemblies, a result of March and Young [11] for spin-free electrons is stressed, and exemplified for a 4-electron model 'atom' solved analytically by Bruch [12] (see also [13]). Then, the third topic selected for this review, namely the equation of motion of the exact correlated 1DM, $\gamma\left(\mathbf{r}, \mathbf{r}^{\prime}\right)$, is set out and illustrated on $\mathrm{He}$ and also on crystalline $\mathrm{Si}$, which completes Sec. 5. Sec. 6 constitutes a summary, plus a note on possible future directions which should prove fruitful. 


\section{Local energy equation in terms of low-order DMs}

The purpose of this section is to summarize the so-called local energy equation [5] (see also references therein) in terms of low-order DMs. But first let us remind the reader of Löwdin's definition $[1,2,3]$ specifically of the second-order density matrix $\Gamma$ from a normalized antisymmetric wave-function $\Psi$. Thus

$$
\Gamma\left(\mathbf{r}_{1}, \mathbf{r}_{2} ; \mathbf{r}_{1}^{\prime}, \mathbf{r}_{2}^{\prime}\right)=\frac{N(N-1)}{2} \int \Psi\left(\mathbf{r}_{1}, \mathbf{r}_{2}, \mathbf{r}_{3} \ldots \mathbf{r}_{N}\right) \Psi^{*}\left(\mathbf{r}_{1}^{\prime}, \mathbf{r}_{2}^{\prime}, \mathbf{r}_{3} \ldots \mathbf{r}_{N}\right) d \mathbf{r}_{3} \ldots d \mathbf{r}_{N},
$$

where $N$ denotes the total number of electrons. The other explicit definition we give is the $1 \mathrm{DM} \gamma$, namely

$$
\gamma\left(\mathbf{r}_{1}, \mathbf{r}_{1}^{\prime}\right)=N \int \Psi\left(\mathbf{r}_{1}, \mathbf{r}_{2} \ldots \mathbf{r}_{N}\right) \Psi^{*}\left(\mathbf{r}_{1}^{\prime}, \mathbf{r}_{2} \ldots \mathbf{r}_{N}\right) d \mathbf{r}_{2} \ldots d \mathbf{r}_{N}
$$

Then, the local energy equation with energy $E$, most typically in this article for the ground state, reads, purely as a consequence of the many-electron Schrödinger equation $[5]$

$$
\begin{aligned}
E \gamma\left(\mathbf{r}, \mathbf{r}^{\prime}\right)= & -\frac{\hbar^{2}}{2 m} \nabla_{\mathbf{r}}^{2} \gamma(\mathbf{r}, \mathbf{r})+V_{\mathrm{ext}}(\mathbf{r}) \gamma\left(\mathbf{r}^{\prime}, \mathbf{r}^{\prime}\right) \\
& +\int u\left(r_{12}\right) \Gamma\left(\mathbf{r}, \mathbf{r}_{2} ; \mathbf{r}^{\prime}, \mathbf{r}_{2}\right) d \mathbf{r}_{2} \\
& +V_{\text {ext }}(\mathbf{r}) \int \Gamma\left(\mathbf{r}, \mathbf{r}_{2} ; \mathbf{r}^{\prime}, \mathbf{r}_{2}\right) d \mathbf{r}_{2} \\
& -\frac{\hbar^{2}}{2 m} \int \nabla_{\mathbf{r}_{2}}^{2} \Gamma\left(\mathbf{r}, \mathbf{r}_{2} ; \mathbf{r}^{\prime}, \mathbf{r}_{2}\right) d \mathbf{r}_{2} \\
& +\frac{1}{2} \int u\left(\left|\mathbf{r}_{3}-\mathbf{r}_{2}\right|\right) \Gamma^{(3)}\left(\mathbf{r}, \mathbf{r}_{2}, \mathbf{r}_{3} ; \mathbf{r}^{\prime}, \mathbf{r}_{2}, \mathbf{r}_{3}\right) d \mathbf{r}_{2} d \mathbf{r}_{3} .
\end{aligned}
$$

In Eq. (3), $V_{\text {ext }}(\mathbf{r})$ denotes the external potential in the atom, molecule, or cluster under investigation, $u\left(r_{12}\right) \equiv u\left(\left|\mathbf{r}_{1}-\mathbf{r}_{2}\right|\right)$ the electron-electron interaction, while $\Gamma^{(3)}$ is the third-order DM in the Löwdin classification. As we shall mainly below utilize Eq. (3) for two-electron spin compensated atoms, for which of course there is no $\Gamma^{(3)}$ term, we merely note here that this $3 \mathrm{DM}$ is defined by a rather obvious generalization of the $2 \mathrm{DM}$ in Eq. (1).

If we divide Eq. (3) throughout by $\gamma\left(\mathbf{r}, \mathbf{r}^{\prime}\right)$, we find the constant ground-state energy $E$ is made up of a sum of six terms, each of which is usually a function of $\mathbf{r}$ and $\mathbf{r}^{\prime}$. We note here the relation to the current usage of DFT, where the constant chemical potential $\mu$ is written as [4]

$$
\mu=\frac{\delta T_{s}}{\delta \rho(\mathbf{r})}+V(\mathbf{r})
$$


The right-hand side is, as shown, the sum of two $\mathbf{r}$-dependent terms. The first is bypassed in Slater-Kohn-Sham (SKS) theory [4], since the single-particle kinetic energy functional $T_{s}[\rho]$ remains unknown, by solving one-body Schrödinger equations with potential energy $V(\mathbf{r})$. Unfortunately, this quantity has an exchange $(x)$ and correlation $(c)$ contribution $V_{x c}(\mathbf{r})$ which remains unknown [4].

It is relevant in this context to mention, as leading into Sec. 3 below, the so-called Moshinsky [6] spin-compensated two-electron model atom, in which both $V_{\text {ext }}(\mathbf{r})$ and $u\left(r_{12}\right)$ are taken as harmonic in nature. If $V_{\text {ext }}(\mathbf{r})=\frac{1}{2} k r^{2}$, with $k=1$ for convenience, and $u\left(r_{12}\right)=\frac{1}{2} K r_{12}^{2}$, then one of us [7] a decade ago obtained the 1DM $\gamma\left(\mathbf{r}_{1}, \mathbf{r}_{1}^{\prime}\right)$ in terms of the density $\rho(\mathbf{r})$ as (see also [8])

$$
\frac{\gamma\left(\mathbf{r}_{1}, \mathbf{r}_{1}^{\prime}\right)}{\gamma\left(\mathbf{r}_{0}, \mathbf{r}_{0}\right)}=\left(\frac{\rho(\bar{r})}{\rho\left(r_{0}\right)}\right)^{\alpha^{2} /(2 \alpha-1)}
$$

where $\mathbf{r}_{0}=\frac{1}{2}\left(\mathbf{r}_{1}+\mathbf{r}_{1}^{\prime}\right), \bar{r}=\left[\frac{1}{2}\left(r_{1}^{2}+r_{1}^{\prime 2}\right)\right]^{1 / 2}$, and the 'interaction strength' parameter $\alpha$ is defined as

$$
\alpha=\frac{1}{2}(1+\sqrt{1+2 K}),
$$

which can in fact be related to the density $\rho(r=0)$ by

$$
\alpha^{-1}=2-\pi[\rho(r=0) / 2]^{2 / 3} .
$$

Evidently, therefore, Eq. (5) for the Moshinsky atom relates the 1DM $\gamma\left(\mathbf{r}_{1}, \mathbf{r}_{1}^{\prime}\right)$ to its diagonal density $\rho\left(\mathbf{r}_{1}\right)$.

This leads us now to discuss the work of Holas, Howard, and March [8], who obtained the $1 \mathrm{DM}$ for a model two-electron atom, still with $V_{\text {ext }}(\mathbf{r})$ of harmonic form, but for arbitrary electron-electron interaction $u\left(r_{12}\right)$, which can therefore embrace the important Hookean atom $[9,10]$, for which $u\left(r_{12}\right)=e^{2} / r_{12}$.

\section{Insight from exact solution of this hierarchy, Eq. (3), for spin-compensated two-electron model atoms}

With the choice of external potential as $V_{\text {ext }}(\mathbf{r})=\frac{1}{2} k r^{2}$, but with general interaction $u\left(r_{12}\right)$, the results of Holas, Howard, and March [8] exploit the centre of mass $(\mathrm{cm})$ and relative motion $(\mathrm{rm})$ separation to write the ground-state wave function as

$$
\Psi\left(\mathbf{r}_{1}, \mathbf{r}_{2}\right)=\Psi_{c m}(\mathbf{R}) \Psi_{r m}\left(r_{12}\right),
$$


where $\mathbf{R}=\frac{1}{2}\left(\mathbf{r}_{1}+\mathbf{r}_{2}\right)$, and, again, $r_{12}=\left|\mathbf{r}_{1}-\mathbf{r}_{2}\right|$. Explicitly, the $\mathrm{cm}$ wave-function is given by

$$
\Psi_{c m}(R)=\exp \left(-\frac{1}{2} \frac{R^{2}}{a_{c m}^{2}}\right),
$$

where $a_{c m}=\left(k / 2 m \omega_{0}\right)^{1 / 2}$, and $\omega_{0}^{2}=k / m$. Writing the $r m$ wave-function as $\Psi_{r m}(r)=$ const $\cdot \psi_{r m}(r) / r$, the one-body Schrödinger equation below results:

$$
\left[-\frac{\hbar^{2}}{2 m_{r m}}\left(\frac{d}{d r}\right)^{2}+\frac{1}{2} m_{r m} \omega_{0}^{2} r^{2}+u(r)\right] \psi_{r m}(r)=E_{r m}(r) \psi_{r m}(r) .
$$

Holas et al. [8] obtained $\gamma\left(\mathbf{r}, \mathbf{r}^{\prime}\right)$ exactly by quadrature on $\Psi_{c m}$ and $\psi_{r m}$. However, the form of $\gamma$ is somewhat complex, so below we limit ourselves to reporting $\left.\gamma\left(\mathbf{r}, \mathbf{r}^{\prime}\right)\right|_{\mathbf{r}=\mathbf{r}^{\prime}}=$ $\rho(\mathbf{r})$ explicitly for general $u(r)$ as (see also [14])

$$
\rho(r)=\frac{8}{\sqrt{\pi}} e^{-r^{2} / a_{c m}^{2}} \int_{0}^{\infty} d y y^{2} e^{-y^{2} / 4}\left[\Psi_{r m}\left(a_{c m} y\right)\right]^{2} \frac{\sinh \left(r y / a_{c m}\right)}{r y / a_{c m}} .
$$

For the Hookean atom, $\rho(r)$ is known analytically for force constant $k=\frac{1}{4}$ a.u. [10]. Though we have not, to date, evaluated the integral in Eq. (11) for this case, Fig. 1 in Ref. [8] shows very good agreement between the numerically evaluated Eq. (11) and the analytical result of Ref. [10]. As shown by [15], the transform entering Eq. (11) can be inverted analytically, if the so-called atomic scattering factor $f(\mathbf{G})$ is introduced, defined by

$$
f(\mathbf{G})=\int \rho(\mathbf{r}) \exp (i \mathbf{G} \cdot \mathbf{r}) d \mathbf{r} .
$$

The result of the inversion of Eq. (11) is then $[15,16]$

$$
\Psi_{r m}^{2}(r)=\frac{1}{16 \pi^{3}} \int e^{G^{2} a_{c m}^{2} / 4} f(\mathbf{G}) e^{-i \mathbf{G} \cdot \mathbf{r} / 2} d \mathbf{G} .
$$

We now employ Eq. (13) to evaluate the total kinetic energy $T$, using the Holas et al. [8] expression

$$
T=\frac{\hbar^{2}}{4 m} \int\left[\frac{d}{d r} \Psi_{c m}(r)\right]^{2} d \mathbf{r}+\frac{\hbar^{2}}{m} \int\left[\frac{d}{d r} \Psi_{r m}(r)\right]^{2} d \mathbf{r},
$$

which, of course, in the language of DFT, includes correlation kinetic energy. Using Eq. (9), the first term on the right-hand side of Eq. (14) is simply $\hbar \omega_{0} / 4$, where $\omega_{0}^{2}=k / m$. The second term, involving arbitrary interaction $u\left(r_{12}\right)$, has been evaluated subsequently by Amovilli and March [16] in the explicit form

$$
T_{r m}=\int t_{r m}(r) d \mathbf{r}
$$


where $t_{r m}(r)$ is given in Eq. (11) of [16] by

$$
t_{r m}(r)=\frac{\hbar^{2}}{64 \pi^{2} m} \frac{\left[\int_{0}^{\infty} G^{3} j_{1}(G r / 2) e^{G^{2} a_{c m}^{2} / 4} f(G) d G\right]^{2}}{\int_{0}^{\infty} G^{2} j_{0}(G r / 2) e^{G^{2} a_{c m}^{2} / 4} f(G) d G},
$$

where $j_{0}$ and $j_{1}$ are spherical Bessel functions. Hence, the total correlated kinetic energy is known for the Holas et al. [8] model for arbitrary interaction $u\left(r_{12}\right)$, given the Fourier transform $f(\mathbf{G})$ of the (known) ground-state density $\rho(r)$ in Eq. (11).

Amovilli and March [16] finally write the total ground-state energy $E$ of this model in terms of $f(\mathbf{G})$ as

$$
E=\frac{3}{2} \hbar \omega_{0}+\frac{\hbar^{2}}{m} \int\left[\frac{d \Psi_{c m}}{d r}\right]^{2} d \mathbf{r}+\int\left[\frac{1}{4} m \omega_{0}^{2} r^{2}+u(r)\right] \Psi_{r m}^{2}(r) d \mathbf{r},
$$

and hence, using Eq. (13) in Eq. (17), $E$ is obtained in terms of the scattering factor $f(\mathbf{G})$.

\section{A variationally valid 1DM for spinless fermions}

More than five decades ago, March and Young [11] made a specific 'variational' proposal for $N$ spinless Fermions as follows. They considered the function $\Phi$ defined by

$$
\Phi\left(\mathbf{r}_{1}, \mathbf{r}_{2} \ldots \mathbf{r}_{N}\right)=\left(\frac{N}{\gamma\left(\mathbf{r}_{1}, \mathbf{r}_{1}\right)}\right)^{1 / 2} \Psi\left(\mathbf{r}_{1}, \mathbf{r}_{2} \ldots \mathbf{r}_{N}\right)
$$

where $\Psi$ is any normalized antisymmetric wave-function. Then, it is easily shown that

$$
\int \Phi^{*}\left(\mathbf{r}_{1}, \mathbf{r}_{2} \ldots \mathbf{r}_{N}\right) \Phi\left(\mathbf{r}_{1}, \mathbf{r}_{2} \ldots \mathbf{r}_{N}\right) d \mathbf{r}_{2} \ldots d \mathbf{r}_{N}=1,
$$

where it must be stressed that there is no integration over $\mathbf{r}_{1}$. Furthermore, $\Phi$ is antisymmetrical in $\mathbf{r}_{2}, \ldots \mathbf{r}_{N}$, and can therefore be utilized to construct DMs of various orders for $N-1$ Fermions in coordinates $\mathbf{r}_{2} \ldots \mathbf{r}_{N}$. In particular,

$$
\frac{2}{\gamma\left(\mathbf{r}_{1}, \mathbf{r}_{1}\right)} \Gamma\left(\mathbf{r}_{1}, \mathbf{r}_{2}^{\prime} ; \mathbf{r}_{1}, \mathbf{r}_{2}\right)
$$

is a variationally valid $1 \mathrm{DM}$ for $N-1$ particles, for arbitrary fixed $\mathbf{r}_{1}$. This latter quantity can therefore be viewed as a 'variational' set of parameters. 


\subsection{Model of low-order density matrices for four electrons in a quintet state}

As an exact example to illustrate the above construction of a variationally valid 1DM, we next note that Bruch [12], some three decades ago, calculated analytically the wavefunction for a model in which four Fermions of spin $\frac{1}{2}$ are mutually interacting with pairwise harmonic attractive forces. But Bruch's solution applied in the limit as the confining potential was allowed to tend to zero.

For the four 'electrons' in the quintet spin state, Akbari, March, and Rubio [13] evaluated the $2 \mathrm{DM} \Gamma$ from the appropriate Bruch wave-function. Their result takes the unnormalized form

$$
\begin{aligned}
\Gamma\left(\mathbf{r}_{1}, \mathbf{r}_{2} ; \mathbf{r}_{1}^{\prime}, \mathbf{r}_{2}^{\prime}\right)= & {\left[\left(\frac{4 \hbar}{m \omega}-\left|\mathbf{S}-\mathbf{S}^{\prime}\right|^{2}\right)\left(\mathbf{r} \cdot \mathbf{r}^{\prime}\right)\right.} \\
& +(\mathbf{S} \cdot \mathbf{r})\left(\mathbf{S} \cdot \mathbf{r}^{\prime}\right)+\left(\mathbf{S}^{\prime} \cdot \mathbf{r}^{\prime}\right)\left(\mathbf{S}^{\prime} \cdot \mathbf{r}\right) \\
& \left.-\left(\mathbf{S}^{\prime} \cdot \mathbf{r}\right)\left(\mathbf{S} \cdot \mathbf{r}^{\prime}\right)\left(\mathbf{S} \cdot \mathbf{r}^{\prime}\right)-(\mathbf{S} \cdot \mathbf{r})\left(\mathbf{S}^{\prime} \cdot \mathbf{r}^{\prime}\right)\right] \\
& \times \exp \left[-\frac{m \omega}{4 \hbar}\left(\left|\mathbf{S}-\mathbf{S}^{\prime}\right|^{2}+r^{2}+r^{\prime 2}\right)\right]
\end{aligned}
$$

Here, $\omega^{2}=K / m$, where $K$ measures the strength of the pairwise attractive harmonic interactions, while $\mathbf{S}=\frac{1}{2}\left(\mathbf{r}_{1}+\mathbf{r}_{2}\right)$, and $\mathbf{r}=\mathbf{r}_{1}-\mathbf{r}_{2}$.

This is now the point at which to return to the March-Young (MY) result referred to above. The MY procedure led to a variational 1DM for $N-1$ spin-free Fermions, i.e. for three parallel spin electrons using Eq. 18 as the starting point.

For compactness below, we merely quote the diagonal element of the resultant 1DM, this being denoted below by $n^{(3)}$, and when written in unnormalized form is given by

$$
n^{(3)}\left(\mathbf{r}_{2}, \mathbf{r}_{1}\right)=\left(\mathbf{r}_{2}-\mathbf{r}_{1}\right)^{2} \exp \left(-\frac{m \omega}{2 \hbar}\left(\mathbf{r}_{2}-\mathbf{r}_{1}\right)^{2}\right),
$$

for arbitrary fixed $\mathbf{r}_{1}$. Consequences of this density are given in [13], to which account the interested reader is referred for further details. The known off-diagonal generalization of Eq. (22) is a variationally valid 1DM, from which the appropriate approximation to the total correlated kinetic energy density $t_{g}$ can be calculated using

$$
t_{g}(\mathbf{r})=\left.\frac{\hbar^{2}}{2 m} \nabla_{\mathbf{r}} \cdot \nabla_{\mathbf{r}^{\prime}} \gamma\left(\mathbf{r}, \mathbf{r}^{\prime}\right)\right|_{\mathbf{r}=\mathbf{r}^{\prime}}
$$




\section{Generalized equation of motion of first-order density matrix}

As the third topic of this review, we shall consider the generalized equation of motion satisfied by the exact correlated ground-state 1DM of a spin-compensated many-electron assembly.

In very early work by March and Young [17], the single-particle $(s)$ 1DM, namely $\gamma_{s}\left(\mathbf{r}, \mathbf{r}^{\prime}\right)$, was calculated using a one-body potential $V(\mathbf{r})$ which generated the orbitals, $\phi_{i}(\mathbf{r})$ say, of the single determinantal wave-function underlying $\gamma_{s}\left(\mathbf{r}, \mathbf{r}^{\prime}\right)$. As was pointed out in [17], the so-called equation of motion satisfied by the idempotent $1 \mathrm{DM} \gamma_{s}\left(\mathbf{r}, \mathbf{r}^{\prime}\right)$ takes the form

$$
\left(\nabla_{\mathbf{r}}^{2}-\nabla_{\mathbf{r}^{\prime}}^{2}\right) \gamma_{s}\left(\mathbf{r}, \mathbf{r}^{\prime}\right)=\frac{2 m}{\hbar^{2}}\left[V(\mathbf{r})-V\left(\mathbf{r}^{\prime}\right)\right] \gamma_{s}\left(\mathbf{r}, \mathbf{r}^{\prime}\right),
$$

which is readily verified to result from the one-body Schrödinger equation with potential energy $V(\mathbf{r})$.

Following Amovilli and March [18], we next seek the generalization of the singleparticle Eq. (24) for the correlated 1DM $\gamma\left(\mathbf{r}, \mathbf{r}^{\prime}\right)$. The above authors solved this problem by expanding $\gamma\left(\mathbf{r}, \mathbf{r}^{\prime}\right)$ in terms of the complete set of orbitals $\phi_{i}(\mathbf{r})$ generated by the one-body potential of DFT, given by [4]

$$
V(\mathbf{r})=V_{\text {Hartree }}(\mathbf{r})+V_{x c}(\mathbf{r}),
$$

where, although as yet unknown, $V_{x c}(\mathbf{r})=\delta E_{x c}[\rho] / \delta \rho(\mathbf{r})$, where $E_{x c}$ denotes the exchange and correlation energy functional [4]. Then, forming the left-hand side of Eq. (24), but with the correlated $\gamma$ given by

$$
\gamma\left(\mathbf{r}, \mathbf{r}^{\prime}\right)=\sum_{\text {all } i, j} n_{i j} \phi_{i}(\mathbf{r}) \phi_{j}^{*}\left(\mathbf{r}^{\prime}\right),
$$

one finds almost immediately

$$
\left(\nabla_{\mathbf{r}}^{2}-\nabla_{\mathbf{r}^{\prime}}^{2}\right) \gamma\left(\mathbf{r}, \mathbf{r}^{\prime}\right)=\frac{2 m}{\hbar^{2}}\left[V(\mathbf{r})-V\left(\mathbf{r}^{\prime}\right)\right] \gamma\left(\mathbf{r}, \mathbf{r}^{\prime}\right)-\frac{2 m}{\hbar^{2}} \sum_{i j} n_{i j}\left(\epsilon_{i}-\epsilon_{j}\right) \phi_{i}(\mathbf{r}) \phi_{j}^{*}\left(\mathbf{r}^{\prime}\right),
$$

$\epsilon_{i}$ denoting the eigenvalue generated by the one-body potential $V$. Using Eq. (24), one readily finds that

$$
\frac{\nabla_{\mathbf{r}}^{2} \gamma-\nabla_{\mathbf{r}^{\prime}}^{2} \gamma}{\gamma}=\frac{\nabla_{\mathbf{r}}^{2} \gamma_{s}-\nabla_{\mathbf{r}^{\prime}}^{2} \gamma_{s}}{\gamma_{s}}-\frac{1}{\gamma} \frac{2 m}{\hbar^{2}} \sum_{i j} n_{i j}\left(\epsilon_{i}-\epsilon_{j}\right) \phi_{i}(\mathbf{r}) \phi_{j}^{*}\left(\mathbf{r}^{\prime}\right)
$$

The attractive feature of Eq. (28), and also of Eq. (27), is that only the off-diagonal elements $n_{i j}$ appearing in the expansion Eq. (26) remain in the summation, because of the presence of the eigenvalue difference $\left(\epsilon_{j}-\epsilon_{i}\right)$. 
To exemplify the usefulness of this 'generalized equation of motion' for the correlated 1DM, Amovilli and March [18] report calculations, albeit of course approximate, of the correlated $1 \mathrm{DM}$ for the ground-state of the He atom, and also for the case of crystalline $\mathrm{Si}$, using results of Kent et al. [19]. These authors recorded quantum Monte Carlo results of the correlated $1 \mathrm{DM}$ for crystalline $\mathrm{Si}$. The interested reader is referred to especially Fig. 3 of Ref. [18], which makes contact with the results of Kent et al. [19].

We next note that following the equation of motion of the 1DM written alternatively in Eqs. (27) and (28), Dawson and March [5] had already derived an alternative form involving directly the 2DM. This reads

$$
\begin{aligned}
&-\frac{\hbar^{2}}{2 m}\left(\nabla_{\mathbf{r}}^{2}-\nabla_{\mathbf{r}^{\prime}}^{2}\right) \gamma\left(\mathbf{r}, \mathbf{r}^{\prime}\right)+ {\left[V_{\mathrm{ext}}(\mathbf{r})-V_{\mathrm{ext}}\left(\mathbf{r}^{\prime}\right)\right] \gamma\left(\mathbf{r}, \mathbf{r}^{\prime}\right) } \\
&+\int\left[u\left(\left|\mathbf{r}-\mathbf{r}_{2}\right|\right)-u\left(\left|\mathbf{r}^{\prime}-\mathbf{r}_{2}\right|\right)\right] \Gamma\left(\mathbf{r}, \mathbf{r}_{2} ; \mathbf{r}^{\prime}, \mathbf{r}_{2}\right) d \mathbf{r}_{2}=0 .
\end{aligned}
$$

For $V_{\text {ext }}(\mathbf{r}) \propto r^{2}$, Holas, Howard, and March [8] have essentially obtained an exact solution of this form of the equation of motion for model two-electron atoms with arbitrary interaction $u\left(r_{12}\right)$, as reported above in Sec. 3 .

\section{Summary and future directions}

After some introductory remarks, plus definitions, attention has been focussed, first of all, on the local energy, Eq. (3), in terms of low-order DMs. It must be emphasized (see [5] especially) that this hierarchical equation is an exact consequence of the manyelectron Schrödinger equation, and is therefore valid for atoms, molecules, and clusters.

But to date, progress in exact analytic form has only proved possible for 2 and 4 electron model atoms. Here, for the 2 electron spin-compensated case, an exact 1DM theory [8] is reviewed for (a) harmonic confinement, and (b) arbitrary interparticle potential energy $u\left(r_{12}\right)$. Eq. (16) completes this theory [20] by obtaining explicitly the total correlated kinetic energy for arbitrary $u\left(r_{12}\right)$ in terms of the Fourier transform $f(\mathbf{G})$ of the ground-state density $\rho(\mathbf{r})$. Eq. (17) gives the ground-state energy in terms of $f(\mathbf{G})$ for this model.

More briefly, in section 4, some progress [13] on the so-called $N$-representability problem is reported using a model 4-electron atom which Bruch [12] has solved exactly. This is transformed, using [11], into a three-electron parallel spin 1DM, which has proven variational validity. Section 5 reports two approaches to an equation of motion of the 1DM, which leads naturally into future directions for fruitful exploration. The final comment then concerns a return to Coulomb confinement. Here, we stress the potential of the two-electron Hamiltonian for a model atomic ion with $V_{\text {ext }}(\mathbf{r})=-Z e^{2} / r$, but with an interaction modified from $u\left(r_{12}\right)=e^{2} / r_{12}$. While this involves the angle $\theta$ between 
vectors $\mathbf{r}_{1}$ and $\mathbf{r}_{2}$, Amovilli, Howard, and March [21] have proposed $u$ involving only scalar $r_{1}, r_{2}$, and no angle $\theta$. The first part of the two term $u\left(r_{1}, r_{2}\right)$ form is due to Temkin [22], while the second involves the addition of the $\delta$-function $\delta\left(r_{1}-r_{2}\right)$. But for this Hamiltonian the ground-state wave-function is known from the earlier work of Howard and March [20]. We firmly believe that further important analytic progress should be possible on this model, which treats Coulomb confinement exactly.

\section{Acknowledgements}

NHM wishes to acknowledge that his contribution to the present review was brought to fruition during a visit to the University of Catania. NHM thanks Professors R. Pucci and G. G. N. Angilella for their kind hospitality.

\section{References}

[1] P. Löwdin (1955). Phys. Rev., 97, 1474.

[2] P. Löwdin (1955). Phys. Rev., 97, 1490.

[3] P. Löwdin (1955). Phys. Rev., 97, 1509.

[4] R. G. Parr and W. Yang, Density Functional Theory of Atoms and Molecules (Oxford University Press, Oxford, 1989).

[5] K. A. Dawson and N. H. March (1984). J. Chem. Phys., 81, 5850. Reprinted in Ref. [23].

[6] M. Moshinsky (1952). Am. J. Phys., 36, 52.

[7] N. H. March (2002). Phys. Lett. A, 306, 63.

[8] A. Holas, I. A. Howard and N. H. March (2003). Phys. Lett. A, 310, 451.

[9] N. R. Kestner and O. Sinanoğlu (1962). Phys. Rev., 128, 2687.

[10] S. Kais, D. R. Herschbach and R. D. Levine (1989). J. Chem. Phys., 91, 7791.

[11] N. H. March and W. H. Young (1959). Phil. Mag, 4, 384.

[12] L. W. Bruch (1980). J. Chem. Phys., 72, 5511.

[13] A. Akbari, N. H. March and A. Rubio (2012). J. Math. Chem., to appear, ...

[14] N. H. March, G. G. N. Angilella and R. Pucci (2012). J. Math. Chem., 50, 914.

[15] A. Akbari, N. H. March and A. Rubio (2009). Phys. Rev. A, 80, 032509.

[16] C. Amovilli and N. H. March (2012). Phys. Scr., 85(6), 065303.

[17] N. H. March and W. H. Young (1959). Nucl. Phys., 12, 237. Reprinted in Ref. [23]. 
[18] C. Amovilli and N. H. March (2012). Phys. Chem. Liq., 50, 121.

[19] P. R. C. Kent, R. Q. Hood, M. D. Towler, R. J. Needs and G. Rajagopal (1998). Phys. Rev. B, 57, 15293.

[20] I. A. Howard and N. H. March (2005). Phys. Rev. A, 71, 042501.

[21] C. Amovilli, I. A. Howard and N. H. March (2008). Phys. Chem. Liq., 46, 238.

[22] A. Temkin (1962). Phys. Rev., 126, 130.

[23] N. H. March and G. G. N. Angilella (Eds.) Many-body Theory of Molecules, Clusters, and Condensed Phases (World Scientific, Singapore, 2009). 